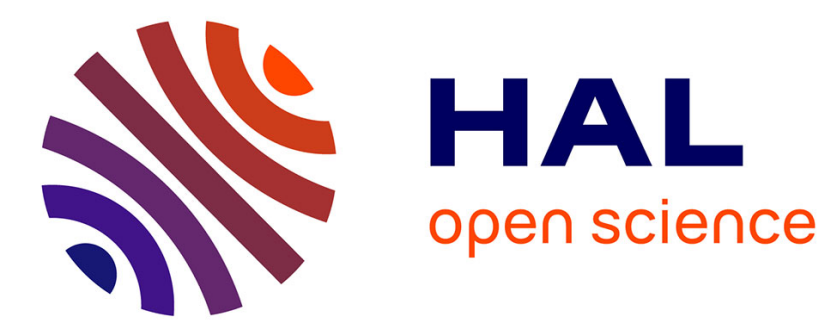

\title{
Error bounds on complex floating-point multiplication with an FMA
}

Claude-Pierre Jeannerod, Peter Kornerup, Nicolas Louvet, Jean-Michel Muller

\section{To cite this version:}

Claude-Pierre Jeannerod, Peter Kornerup, Nicolas Louvet, Jean-Michel Muller. Error bounds on complex floating-point multiplication with an FMA. 2013. hal-00867040v1

\section{HAL Id: hal-00867040 https://hal.inria.fr/hal-00867040v1}

Preprint submitted on 27 Sep 2013 (v1), last revised 4 Jan 2017 (v5)

HAL is a multi-disciplinary open access archive for the deposit and dissemination of scientific research documents, whether they are published or not. The documents may come from teaching and research institutions in France or abroad, or from public or private research centers.
L'archive ouverte pluridisciplinaire HAL, est destinée au dépôt et à la diffusion de documents scientifiques de niveau recherche, publiés ou non, émanant des établissements d'enseignement et de recherche français ou étrangers, des laboratoires publics ou privés. 


\title{
ERROR BOUNDS ON COMPLEX FLOATING-POINT MULTIPLICATION WITH AN FMA
}

\author{
CLAUDE-PIERRE JEANNEROD, PETER KORNERUP, NICOLAS LOUVET, \\ AND JEAN-MICHEL MULLER
}

\begin{abstract}
The accuracy analysis of complex floating-point multiplication done by Brent, Percival, and Zimmermann [Math. Comp., 76:1469-1481, 2007] is extended to the case where a fused multiply-add (FMA) operation is available. Considering floating-point arithmetic with rounding to nearest and unit roundoff $u$, we show that their bound $\sqrt{5} u$ on the normwise relative error $|\widehat{z} / z-1|$ of a complex product $z$ can be decreased further to $2 u$ when using the FMA in the most naive way. Furthermore, we prove that the term $2 u$ is asymptotically optimal not only for this naive FMA-based algorithm, but also for two other algorithms, which use the FMA operation as an efficient way of implementing rounding error compensation. Thus, although highly accurate in the componentwise sense, these two compensated algorithms bring no improvement to the normwise accuracy $2 u$ already achieved using the FMA naively. Asymptotic optimality is established for each algorithm thanks to the explicit construction of floating-point inputs for which we prove that the normwise relative error then generated satisfies $|\widehat{z} / z-1| \rightarrow 2 u$ as $u \rightarrow 0$. All our results hold for IEEE floating-point arithmetic, with radix $\beta \geqslant 2$, precision $p \geqslant 2$, and rounding to nearest; it is only assumed that underflows and overflows do not occur and, when bounding errors from below, that $\beta^{p-1} \geqslant 12$.
\end{abstract}

\section{INTRODUCTION}

Given complex numbers $x=a+i b$ and $y=c+i d$, let their product $z=x y$ be expressed as

$$
z=a c-b d+i(a d+b c) .
$$

Assuming that $a, b, c, d$ are floating-point numbers and that the operations,,$+- \times$ on such numbers are performed with rounding to nearest (RN), the conventional way of evaluating the expression above can be described as follows:

$$
\mathcal{A}_{0}:(a+i b, c+i d) \mapsto \mathrm{RN}(\mathrm{RN}(a c)-\mathrm{RN}(b d))+i \cdot \mathrm{RN}(\mathrm{RN}(a d)+\mathrm{RN}(b c))
$$

The normwise accuracy of algorithm $\mathcal{A}_{0}$ was studied by Brent, Percival, and Zimmermann [3] for standard floating-point arithmetic (with radix $\beta$, precision $p$, and rounding to nearest) and assuming that underflows and overflows do not occur. They showed that the computed value has the form

$$
\widehat{z}_{0}=z(1+\epsilon), \quad|\epsilon|<\sqrt{5} u, \quad u=\frac{1}{2} \beta^{1-p},
$$

September 27, 2013.

2010 Mathematics Subject Classification. Primary 65G50. 
which for $z$ nonzero implies that the normwise relative error $\left|\widehat{z}_{0} / z-1\right|$ is always less than $\sqrt{5}=2.236 \ldots$ times the unit roundoff. For $\beta=2$ and rounding 'to nearest even' they also showed by constructing specific inputs for $\mathcal{A}_{0}$ that the upper bound $\sqrt{5} u$ should be considered sharp: in the cases $p=24$ and $p=53$ the largest possible errors have the form $\sqrt{4.9999899864 \ldots u} u$ and $\sqrt{4.9999999999999893 \ldots u} u$, respectively; more generally, when $p \geqslant 2$ they provide floating-point numbers $a, b, c, d$ for which $\left|\widehat{z}_{0} / z-1\right|=\sqrt{5} u-O\left(u^{2}\right)$ as $u \rightarrow 0$, so that the relative error bound $\sqrt{5} u$ is asymptotically optimal for algorithm $\mathcal{A}_{0}$.

The goal of our paper is to extend this study of the normwise accuracy of complex floating-point multiplication by allowing not only floating-point,,$+- \times$ but also the fused multiply-add (FMA) operation. Given three floating-point numbers $a, b, c$ the FMA evaluates expressions of the form $a b+c$ with one rounding error instead of two, so that with round to nearest, the result is $\mathrm{RN}(a b+c)$ rather than $\mathrm{RN}(\mathrm{RN}(a b)+c)$. This operation has been required since the 2008 revision of the IEEE 754 standard for floating-point arithmetic [7], and is therefore being supported by an increasing number of processors.

With an FMA, the conventional way of evaluating $a c-b d+i(a d+b c)$ becomes:

$$
\mathcal{A}_{1}:(a+i b, c+i d) \mapsto \mathrm{RN}(a c-\mathrm{RN}(b d))+i \cdot \mathrm{RN}(a d+\mathrm{RN}(b c))
$$

Algorithm $\mathcal{A}_{1}$ is of course just one of four variants that differ only in the choice of the products to which the FMA operations apply. Our first contribution is to prove that for any of these four conventional FMA-based algorithms the computed complex product $\widehat{z}_{1}$ satisfies

$$
\left|\widehat{z}_{1}-z\right| \leqslant 2 u|z|
$$

and, by constructing inputs $a, b, c, d$ for which $\left|\widehat{z}_{1} / z-1\right|=2 u-O\left(u^{1.5}\right)$ as $u \rightarrow 0$, that the relative error bound (1.1) is asymptotically optimal.

Another classical way of exploiting the FMA is for efficiently computing the error in a floating-point product $[15, \S 4.4]$ : given two floating-point numbers $a$ and $b$ and barring underflow and overflow, the error $e=a b-\mathrm{RN}(a b)$ can be produced as

$$
e=\mathrm{RN}(a b-\mathrm{RN}(a b))
$$

in one multiplication and one FMA. (In contrast, without an FMA and using only ,,$+- \times$, the cheapest algorithm we are aware of is due to Dekker and Veltkamp and uses 17 operations; see [5, 2] and [15, p. 135].) Once such rounding errors $e$ are available, they can be used to construct a correction term aimed at improving the overall accuracy of the computed result. This approach, called compensation, can be traced back to the works of Møller [13, 12], Kahan [9], Dekker [5], Pichat [16, 17], and Linnainmaa [10, 11]; Cornea, Harrison, and Tang [4, p. 273] use it explicitly in the following algorithm to evaluate

$$
r=a b+c d
$$

accurately in 7 floating-point operations: 


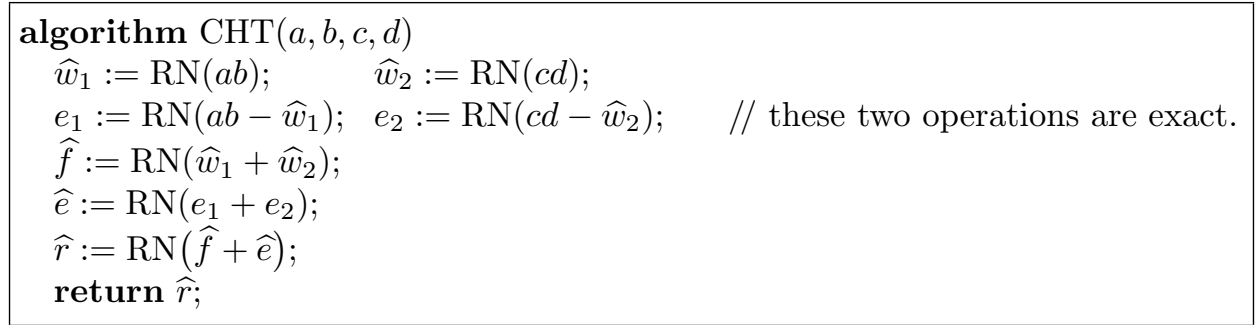

In algorithm CHT an approximation $\widehat{f}$ to $r$ is computed by simply evaluating and adding the products $a b$ and $c d$. Simultaneously, the rounding errors $e_{1}$ and $e_{2}$ due to the evaluation of these two products are computed with two FMA operations, and then added together into a correction term $\widehat{e}$. The corrected solution $\widehat{r}$ is finally produced as the rounded sum of $\widehat{f}$ and $\widehat{e}$. While $\widehat{f}$ can be inaccurate due to cancellation, $\widehat{r}$ turns out to be always highly accurate, at least in radix 2 : Cornea, Harrison, and Tang [4] show that in the absence of underflow and overflow $|\widehat{r}-r| \leqslant$ $O(u)|r|$ and, analyzing their algorithm further, Muller [14] shows that

$$
|\widehat{r}-r| \leqslant\left(2 u+O\left(u^{2}\right)\right)|r| \quad \text { if } \beta=2,
$$

and that this bound is asymptotically optimal.

A straightforward application of the CHT algorithm is to evaluate accurately the real and imaginary parts of the complex product $z=a c-b d+i(a d+b c)$. This is shown in algorithm $\mathcal{A}_{2}$ below, which uses 14 floating-point operations:

$$
\mathcal{A}_{2}:(a+i b, c+i d) \mapsto \operatorname{CHT}(a, c,-b, d)+i \cdot \operatorname{CHT}(a, d, b, c)
$$

By applying (1.2a) twice (first with $r=a c-b d$, and then with $r=a d+b c$ ), we deduce immediately that if $\beta=2$, then the approximate product $\widehat{z}_{2}$ computed by $\mathcal{A}_{2}$ satisfies

$$
\left|\widehat{z}_{2}-z\right| \leqslant\left(2 u+O\left(u^{2}\right)\right)|z| .
$$

In this paper, our second contribution is to show that the bound (1.2b) in fact holds not only for $\beta=2$ but also for all $\beta>2$ independently of (1.2a), and on the other hand, that it is asymptotically optimal for algorithm $\mathcal{A}_{2}$. In particular, this asymptotic optimality result says that the compensation for the errors in $a c$, $b d, a d, b c$ performed by algorithm CHT brings no improvement to the normwise relative accuracy $2 u$ of the non-compensated, conventional algorithm $\mathcal{A}_{1}$.

Algorithm CHT makes no use of the FMA to produce the initial approximation $\widehat{f}$ to $r=a b+c d$. However, if we employ the FMA already at this stage, then the rounding error of only one product (say, $c d$ ) needs to be recovered to eventually ensure high relative accuracy. This is the basis of the algorithm below, attributed to Kahan in [6, p. 65], that evaluates $r$ accurately in 4 floating-point operations:

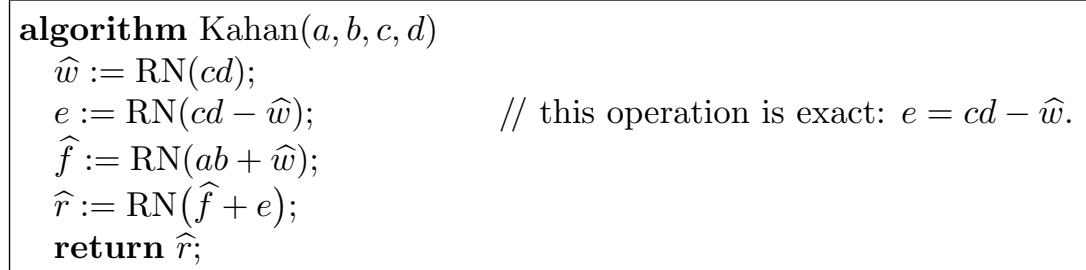


It was shown in [8, Theorem 1.2] that for $\beta, p \geqslant 2$ and in the absence of underflow and overflow the result $\widehat{r}$ produced by Kahan's algorithm satisfies

$$
|\widehat{r}-r| \leqslant 2 u|r|
$$

and, for $\beta$ even, that this relative error bound is asymptotically optimal. In the same way as for the CHT algorithm, we can apply Kahan's algorithm to the accurate evaluation of the real and imaginary parts of the complex product $z$, but now using 8 floating-point operations instead of 14 :

$$
\mathcal{A}_{3}:(a+i b, c+i d) \mapsto \operatorname{Kahan}(a, c,-b, d)+i \cdot \operatorname{Kahan}(a, d, b, c)
$$

Since the expression $r=a b+c d$ can be evaluated either as $\operatorname{Kahan}(a, b, c, d)$ or $\operatorname{Kahan}(c, d, a, b)$, algorithm $\mathcal{A}_{3}$ comes in fact with three other variants. Using (1.3a), we see immediately that for any of these four algorithms the computed complex product $\widehat{z}_{3}$ satisfies

$$
\left|\widehat{z}_{3}-z\right| \leqslant 2 u|z|
$$

Our third contribution in this paper is to show that the normwise relative error bound (1.3b) is asymptotically optimal. Thus, although $\widehat{z}_{3}$ enjoys a slightly sharper bound than $\widehat{z}_{2}$ and is produced at a lower arithmetic cost, it still does not improve on the accuracy achieved by the straightforward FMA-based solution $\widehat{z}_{1}$.

To summarize, the two main conclusions in this paper are as follows:

- The availability of an FMA makes it possible to replace the classical accuracy bound $\sqrt{5} u$ by $2 u$, and this new bound is sharp when the FMA is used in the conventional way, as in algorithm $\mathcal{A}_{1}$;

- The bound $2 u$ cannot be reduced further by FMA-based, compensated schemes like algorithms $\mathcal{A}_{2}$ and $\mathcal{A}_{3}$.

These conclusions hold for normwise relative accuracy only. For componentwise relative accuracy, where we bound $\max (|\operatorname{Re} \widehat{z} / \operatorname{Re} z-1|,|\operatorname{Im} \widehat{z} / \operatorname{Im} z-1|)$ instead of $|\widehat{z} / z-1|$, the benefit of using FMA-based compensation via the CHT algorithm or Kahan's cheaper variant is clear: algorithms $\mathcal{A}_{2}$ and $\mathcal{A}_{3}$ guarantee a tiny componentwise relative error, while algorithms $\mathcal{A}_{0}$ and $\mathcal{A}_{1}$ can both be highly inaccurate due to possible catastrophic cancellations in the real or imaginary part of the computed product.

This paper is organized as follows. After some preliminaries in Section 2, we establish the normwise relative error bounds (1.1) and (1.2b) in Sections 3.1 and 3.2, respectively. Section 4 then gathers our asymptotic optimality results: we begin, in Section 4.1, by constructing some input for which both algorithm $\mathcal{A}_{1}$ and algorithm $\mathcal{A}_{3}$ have their normwise relative error lower bounded by $2 u-O\left(u^{1.5}\right)$; in Section 4.2, this lower bound is achieved for both algorithm $\mathcal{A}_{2}$ and algorithm CHT via the construction of another input. Concluding remarks are given in Section 5.

Finally, let us emphasize that all the error bounds presented in this paper only assume the absence of underflow/overflow as well as some mild assumptions on the radix $\beta$ and precision $p$ : all our upper bounds are valid for $\beta \geqslant 2$ and $p \geqslant 2$, and all our lower bounds assume $\beta^{p-1} \geqslant 12$. Therefore, the results of this paper hold for all IEEE 754-2008 floating-point formats and as long as underflows and overflows do not occur. 


\section{Preliminaries}

This section provides the main definitions and assumptions used in the paper. We also show that when analyzing the normwise accuracy of any of the complex multiplication algorithms studied here, we can assume with no loss of generality that the operands $a+i b$ and $c+i d$ satisfy $a b c d \geqslant 0$. Then, we consider the possible variants of algorithms $\mathcal{A}_{1}$ and $\mathcal{A}_{3}$, and show that we can restrict the accuracy analyses to these two algorithms only. Finally, we recall that $(1.3 \mathrm{~b})$ is simply a consequence of $[8$, Theorem 1.2].

2.1. Definitions and assumptions. Throughout this paper $\beta$ and $p$ are integers such that

$$
\beta, p \geqslant 2,
$$

and $\mathbb{F}$ is the set of floating-point numbers with radix $\beta$ and precision $p$, assuming an unbounded exponent range:

$$
\mathbb{F}=\{0\} \cup\left\{M \cdot \beta^{e-p+1}: M, e \in \mathbb{Z}, \beta^{p-1} \leqslant|M|<\beta^{p}\right\} .
$$

Associated with this set are the unit roundoff $u=\frac{1}{2} \beta^{1-p}$ as well as a round-tonearest function $\mathrm{RN}$, which maps any real number $t$ to a nearest element in $\mathbb{F}$, denoted by $\mathrm{RN}(t)$. Note that since all the results in this paper are proved using $\mathbb{F}$, whose range is unbounded, they remain valid for IEEE floating-point arithmetic as long as neither underflow nor overflow occurs.

We write ulp to denote the unit in the last place function: $u l p(0)=0$ and for any nonzero real number $t, \operatorname{ulp}(t)$ is the unique integer power of $\beta$ such that $\beta^{p-1} \leqslant|t| / \mathrm{ulp}(t)<\beta^{p}$. Combining the definitions of RN, ulp, and $u$ leads to

$$
|\mathrm{RN}(t)-t| \leqslant \frac{1}{2} \mathrm{ulp}(t) \leqslant u|t| \text { for any real number } t \text {. }
$$

In particular, it follows that the exact result $t$ of a floating-point operation is related to its correctly-rounded value $\widehat{t}=\mathrm{RN}(t)$ by the identity below, referred to as the standard model of floating-point arithmetic [6, p. 40]:

$$
\widehat{t}=t(1+\delta), \quad|\delta| \leqslant u .
$$

Here and hereafter, $z$ denotes the exact product of two complex numbers $a+i b$ and $c+i d$ having their real and imaginary parts in $\mathbb{F}$, that is,

$$
z=a c-b d+i(a d+b c), \quad a, b, c, d \in \mathbb{F} .
$$

For each of the complex multiplication algorithms introduced in Section 1, we define

$$
\widehat{z}_{h}=\text { the approximation to } z \text { produced by algorithm } \mathcal{A}_{h} \text { for } h \in\{0,1,2,3\},
$$

and, when dealing with the real and imaginary parts of $z$ and $\widehat{z}_{h}$ explicitly, we shall use the notations

$$
z=R+i I, \quad \widehat{z}_{h}=\widehat{R}_{h}+i \widehat{I}_{h} .
$$

Note in particular that replacing $(c, d)$ by $(-d, c)$ changes the sign of the product $a b c d$ but has no effect on the rounding errors committed: the exact and approximate products $z$ and $\widehat{z}_{h}$ become $\zeta=-I+i R$ and $\widehat{\zeta}_{h}=-\widehat{I}_{h}+i \widehat{R}_{h}$, respectively, so that $|\zeta|=|z|$ and $\left|\widehat{\zeta}_{h}-\zeta\right|=\left|\widehat{z}_{h}-z\right|$. Therefore, when establishing our error bounds it will always be possible to assume without loss of generality that

$$
a b c d \geqslant 0 \text {. }
$$


2.2. Variants of algorithms $\mathcal{A}_{1}$ and $\mathcal{A}_{3}$. As already mentioned in introduction, algorithms $\mathcal{A}_{1}$ and $\mathcal{A}_{3}$ have three other variants each. This is due to the fact that with an FMA the expression $a b+c d$ can be evaluated either as $\mathrm{RN}(a b+\mathrm{RN}(c d))$ or as $\mathrm{RN}(\mathrm{RN}(a b)+c d)$, each of these two ways possibly producing a different result.

Given

$$
x=a+i b, \quad y=c+i d,
$$

the complex floating-point numbers returned by algorithm $\mathcal{A}_{1}$ and its variants are displayed in the following table:

\begin{tabular}{cc}
$\ell$ & $\widehat{z}_{1}^{(\ell)}(x, y)$ \\
\hline 1 & $\mathrm{RN}(a c-\mathrm{RN}(b d))+i \cdot \operatorname{RN}(a d+\mathrm{RN}(b c))$ \\
2 & $\mathrm{RN}(a c-\mathrm{RN}(b d))+i \cdot \operatorname{RN}(\mathrm{RN}(a d)+b c)$ \\
3 & $\mathrm{RN}(\mathrm{RN}(a c)-b d)+i \cdot \operatorname{RN}(\mathrm{RN}(a d)+b c)$ \\
4 & $\mathrm{RN}(\mathrm{RN}(a c)-b d)+i \cdot \operatorname{RN}(a d+\mathrm{RN}(b c))$
\end{tabular}

Here, $\widehat{z}_{1}^{(1)}(x, y)$ is the output of algorithm $\mathcal{A}_{1}$. Although the $\widehat{z}_{1}^{(\ell)}(x, y)$ are pairwise distinct for most $(x, y)$, they share the same normwise error bound. To see this, let

$$
\left(x^{(2)}, y^{(2)}\right)=(y, x), \quad\left(x^{(3)}, y^{(3)}\right)=(-i x, i y), \quad\left(x^{(4)}, y^{(4)}\right)=(i y,-i x) .
$$

For $\ell=2,3,4$ we see that $x^{(\ell)} y^{(\ell)}=x y$ and it is easily checked that $\widehat{z}_{1}^{(\ell)}(x, y)=$ $\widehat{z}_{1}^{(1)}\left(x^{(\ell)}, y^{(\ell)}\right)$, thus leading to

$$
\left|\widehat{z}_{1}^{(\ell)}(x, y)-x y\right|=\left|\widehat{z}_{1}^{(1)}\left(x^{(\ell)}, y^{(\ell)}\right)-x^{(\ell)} y^{(\ell)}\right| .
$$

In other words, for $\ell=2,3,4$, the normwise (absolute or relative) error committed when approximating the product $x y$ by $\widehat{z}_{1}^{(\ell)}(x, y)$ is the same as the one committed by algorithm $\mathcal{A}_{1}$ for some input $\left(x^{(\ell)}, y^{(\ell)}\right)$ that can be deduced from $(x, y)$ via the error-free transformations defined in (2.3). Consequently, the four conventional algorithms with FMA enjoy the same normwise error bounds, and we shall focus on the analysis of $\mathcal{A}_{1}$ only.

Similarly, four complex multiplication algorithms based on Kahan's algorithm are also possible:

\begin{tabular}{cc}
$\ell$ & $\widehat{z}_{3}^{(\ell)}(x, y)$ \\
\hline 1 & $\operatorname{Kahan}(a, c,-b, d)+i \cdot \operatorname{Kahan}(a, d, b, c)$ \\
2 & $\operatorname{Kahan}(a, c,-b, d)+i \cdot \operatorname{Kahan}(b, c, a, d)$ \\
3 & $\operatorname{Kahan}(-b, d, a, c)+i \cdot \operatorname{Kahan}(b, c, a, d)$ \\
4 & $\operatorname{Kahan}(-b, d, a, c)+i \cdot \operatorname{Kahan}(a, d, b, c)$
\end{tabular}

The value returned by algorithm $\mathcal{A}_{3}$ is $\widehat{z}_{3}^{(1)}(x, y)$ and, using (2.3) and the same reasoning as before, we can check that it shares the same normwise error bounds as any of $\widehat{z}_{3}^{(\ell)}(x, y), \ell=2,3,4$. Hence it suffices to analyze algorithm $\mathcal{A}_{3}$ to deduce the error behavior of any of its three other variants.

2.3. Error bound for algorithm $\mathcal{A}_{3}$ as a consequence of [8, Theorem 1.2]. We remarked in introduction that the error bound given in (1.3b) for algorithm $\mathcal{A}_{3}$ follows immediately from the error bound (1.3a) obtained in [8, Theorem 1.2] for Kahan's algorithm. For the sake of completeness, we summarize this result in the following theorem: 
Theorem 2.1. For $\beta, p \geqslant 2$ and in the absence of underflow and overflow, algorithm $\mathcal{A}_{3}$ computes $\widehat{z}_{3}$ such that

$$
\left|\widehat{z}_{3}-z\right| \leqslant 2 u|z|
$$

Proof. By definition, algorithm $\mathcal{A}_{3}$ approximates $z=R+i I$ by $\widehat{z}_{3}=\widehat{R}_{3}+i \widehat{I}_{3}$, where, using [8, Theorem 1.2], $\left|\widehat{R}_{3}-R\right| \leqslant 2 u|R|$ and $\left|\widehat{I}_{3}-I\right| \leqslant 2 u|I|$. Hence $\left|\widehat{z}_{3}-z\right|=\sqrt{\left(\widehat{R}_{3}-R\right)^{2}+\left(\widehat{I}_{3}-I\right)^{2}} \leqslant 2 u \sqrt{R^{2}+I^{2}}=2 u|z|$.

\section{Error bounds for algorithms $\mathcal{A}_{1}$ AND $\mathcal{A}_{2}$}

In this section, we show that the relative errors of algorithms $\mathcal{A}_{1}$ and $\mathcal{A}_{2}$ are upper bounded by $2 u$ and $2 u+O\left(u^{2}\right)$, respectively. These bounds hold for any radix and precision $\beta, p \geqslant 2$ and as long as underflows and overflows do not occur.

3.1. Error bound for algorithm $\mathcal{A}_{1}$. The goal of this subsection is to establish the following result:

Theorem 3.1. For $\beta, p \geqslant 2$ and in the absence of underflow and overflow, algorithm $\mathcal{A}_{1}$ computes $\widehat{z}_{1}$ such that

$$
\left|\widehat{z}_{1}-z\right| \leqslant 2 u|z|
$$

To prove this theorem we rely on three lemmas that provide suitable bounds on the absolute errors $\left|\widehat{R}_{1}-R\right|$ and $\left|\widehat{I}_{1}-I\right|$.

Lemma 3.1. $\left|\widehat{R}_{1}-R\right| \leqslant u|R|+u|b d|+u^{2}|b d|$.

Proof. Applying the standard model (2.2) to the real part $\widehat{R}_{1}$ of the result of algorithm $\mathcal{A}_{1}$ gives $\widehat{R}_{1}=\left(a c-b d\left(1+\epsilon_{1}\right)\right)\left(1+\epsilon_{2}\right)$ with $\left|\epsilon_{1}\right|,\left|\epsilon_{2}\right| \leqslant u$. Since $R=a c-b d$, we deduce that $\widehat{R}_{1}-R=R \epsilon_{2}-b d \epsilon_{1}-b d \epsilon_{1} \epsilon_{2}$. The result follows from the triangle inequality and the bounds on $\left|\epsilon_{1}\right|$ and $\left|\epsilon_{2}\right|$.

Similarly, the imaginary part of $\widehat{z}_{1}$ satisfies $\left|\widehat{I}_{1}-I\right| \leqslant u|I|+u|b c|+u^{2}|b c|$. The next two lemmas aim at removing the $O\left(u^{2}\right)$ terms in each of these bounds.

Lemma 3.2. If $a b c d \geqslant 0$ then $\left|\widehat{I}_{1}-I\right| \leqslant u|I|+u|b c|$.

Proof. Recalling that $I=a d+b c$ and $\widehat{I}_{1}=\mathrm{RN}(g)$ with $g=a d+\mathrm{RN}(b c)$, we have

$$
\begin{aligned}
\left|\widehat{I}_{1}-I\right| & \leqslant|\operatorname{RN}(g)-g|+|\operatorname{RN}(b c)-b c| \\
& \leqslant \frac{1}{2} \mathrm{ulp}(g)+u|b c| .
\end{aligned}
$$

Since $\frac{1}{2} \mathrm{ulp}(I) \leqslant u|I|$, the conclusion follows immediately when $u \operatorname{lp}(g) \leqslant \mathrm{ulp}(I)$. Assume now that $\operatorname{ulp}(I)<\mathrm{ulp}(g)$. In this case, $|I|<\beta^{k} \leqslant|g|$ for some integer $k$ and, since $\beta^{k} \in \mathbb{F}$ and by definition of rounding to nearest, $|\operatorname{RN}(g)-g| \leqslant|g|-\beta^{k}<$ $|g|-|I| \leqslant|g-I|=|\operatorname{RN}(b c)-b c| \leqslant u|b c|$. Therefore, $\left|\widehat{I}_{1}-I\right| \leqslant 2 u|b c|$. By assumption, the products $a d$ and $b c$ have the same sign, so that $|b c| \leqslant|I|$ and the conclusion follows.

Lemma 3.3. If $a b c d \geqslant 0$ and $|a c|<\frac{1}{2}|b d|$ then $\left|\widehat{R}_{1}-R\right| \leqslant u|R|+u|b d|$. 
Proof. Let $f=a c-\mathrm{RN}(b d)$ and consider the following condition:

$b d \notin \mathbb{F}$ and $f \notin \mathbb{F}$.

If (C) is not satisfied then either $b d \in \mathbb{F}$, which implies $\left|\widehat{R}_{1}-R\right| \leqslant u|R|$, or $f \in \mathbb{F}$, which implies $\left|\widehat{R}_{1}-R\right| \leqslant u|b d|$. Hence the result is true in this case, and from now we assume that $(\mathrm{C})$ holds. We have

$$
\begin{aligned}
\left|\widehat{R}_{1}-R\right| & \leqslant|\mathrm{RN}(f)-f|+|\mathrm{RN}(b d)-b d| \\
& \leqslant \frac{1}{2} \mathrm{ulp}(f)+u|b d|,
\end{aligned}
$$

so that the result is true as soon as $\operatorname{ulp}(f) \leqslant u l p(R)$.

Assume now that $\operatorname{ulp}(R)<\operatorname{ulp}(f)$, that is, $\operatorname{ulp}(R) \leqslant \beta^{-1} \operatorname{ulp}(f)$. Defining $e=\mathrm{RN}(b d)-b d$, we see that $R=f+e$ and then $|f| \leqslant|R|+|e|$. Now, using [8, Lemma 3.1] gives $|f| \leqslant \beta|R|$. Hence $\operatorname{ulp}(f) \leqslant \beta u l p(R)$ and we conclude that

$$
\operatorname{ulp}(f)=\beta \operatorname{ulp}(R) .
$$

If $\operatorname{ulp}(b d) \leqslant \operatorname{ulp}(R)$ then, by [8, Lemma 5.5] we have $|\operatorname{RN}(f)-f| \leqslant \frac{1}{2} u l p(R)$, from which the result follows.

If $\mathrm{ulp}(b d)>\mathrm{ulp}(R)$ then we have

$$
\operatorname{ulp}(b d)=\beta \operatorname{ulp}(R),
$$

since the assumption $|a c|<\frac{1}{2}|b d|$ implies $|R|=|b d|-|a c|>\frac{1}{2}|b d| \geqslant \frac{1}{\beta}|b d|$ and thus $\mathrm{ulp}(R) \geqslant \beta^{-1} \mathrm{ulp}(b d)$. To summarize, we are left with dealing with the case where

$$
\mathrm{ulp}(b d)=\mathrm{ulp}(f)=\beta \mathrm{ulp}(R) .
$$

In order to handle this case, remark first that

$$
|f|=|\mathrm{RN}(b d)|-|a c| .
$$

Indeed, since $a b c d \geqslant 0$ by assumption, the three quantities $a c, b d$, and $\mathrm{RN}(b d)$ have the same sign, so that $|f|=|| a c|-| \mathrm{RN}(b d)||$; furthermore, since we have also assumed that $|a c|<\frac{1}{2}|b d|$, we have $|\operatorname{RN}(b d)|-|a c| \geqslant(1-u)|b d|-|a c| \geqslant\left(\frac{1}{2}-u\right)|b d| \geqslant$ 0 for all $\beta, p \geqslant 2$.

Remark also that since $\operatorname{ulp}(R)<\operatorname{ulp}(f)$, there exists an integer $k$ such that

$$
|R|<\beta^{k} \leqslant|f| \text {. }
$$

Defining $\delta=\mathrm{ulp}(b d)$, we have $|f| \leqslant|b d|+\frac{1}{2} \mathrm{ulp}(b d)-|a c|=|R|+\frac{\delta}{2}$ and thus

$$
|f|<\beta^{k}+\frac{\delta}{2} \text {. }
$$

Since $\delta$ is also equal to $\frac{1}{2} \operatorname{ulp}(f)$ we deduce that $\left|\widehat{R}_{1}\right|=|\mathrm{RN}(f)|=\mathrm{RN}(|f|)$ satisfies

$$
\left|\widehat{R}_{1}\right|=\beta^{k} .
$$

On the other hand, $|R|=|b d|-|a c| \geqslant|\operatorname{RN}(b d)|-\frac{1}{2} \mathrm{ulp}(b d)-|a c|=|f|-\frac{1}{2} \mathrm{ulp}(b d)$, so that

$$
|R| \geqslant \beta^{k}-\frac{\delta}{2}
$$

To conclude, note that the assumption $|a c|<\frac{1}{2}|b d|$ implies that $R$ and $\widehat{R}_{1}$ have the same sign: indeed, $|R|=|b d|-|a c|>\frac{1}{2}|b d|$, which by Lemma 3.1 implies $\left|\widehat{R}_{1}-R\right|<u(3+2 u)|R|<|R|$ for all $\beta, p \geqslant 2$. Consequently,

$$
\left|\widehat{R}_{1}-R\right|=|| \widehat{R}_{1}|-| R||=\left|\widehat{R}_{1}\right|-|R| \leqslant \beta^{k}-\left(\beta^{k}-\frac{\delta}{2}\right)=\frac{1}{2} \mathrm{ulp}(b d)
$$

and then $\left|\widehat{R}_{1}-R\right| \leqslant u|b d|$. 
Using Lemmas 3.1, 3.2, and 3.3 we can now establish Theorem 3.1 as follows.

Proof of Theorem 3.1. We can assume without loss of generality that abcd $\geqslant 0$. This implies $\operatorname{sign}(a c)=\operatorname{sign}(b d)$, from which it follows that

$$
|R|=|| a c|-| b d|| \text {. }
$$

We now consider separately three cases, depending on how $|a c|$ relates to $|b d|$. In each case we show that $\left|\widehat{z}_{1}-z\right| \leqslant 2 u|z|$ by checking the equivalent inequality

$$
Q \leqslant 4\left(R^{2}+I^{2}\right)
$$

where $Q=\frac{1}{u^{2}}\left(\left(\widehat{R}_{1}-R\right)^{2}+\left(\widehat{I}_{1}-I\right)^{2}\right)$ and $R^{2}+I^{2}=(a c)^{2}+(b d)^{2}+(a d)^{2}+(b c)^{2}$.

If $|a c| \geqslant|b d|$ then $|R|=|a c|-|b d|$, so that Lemmas 3.1 and 3.2 imply

$$
\begin{aligned}
Q & \leqslant(|a c|+u|b d|)^{2}+(|I|+|b c|)^{2} \\
& \leqslant(|a c|+|b d|)^{2}+(|a d|+2|b c|)^{2} \\
& =(a c)^{2}+(b d)^{2}+(a d)^{2}+4(b c)^{2}+6 \pi,
\end{aligned}
$$

where

$$
\pi:=a b c d .
$$

The inequality in (3.1) then follows from $2 \pi \leqslant(a c)^{2}+(b d)^{2}$.

If $\frac{1}{2}|b d| \leqslant|a c|<|b d|$ then $|R|=|b d|-|a c|$ and $\frac{1}{2}(b d)^{2} \leqslant \pi$. Thus, by Lemma 3.2,

$$
\begin{aligned}
Q & \leqslant(2|b d|-|a c|+u|b d|)^{2}+(|a d|+2|b c|)^{2} \\
& =(a c)^{2}+4(b d)^{2}+(a d)^{2}+4(b c)^{2}+Q^{\prime}+Q^{\prime \prime},
\end{aligned}
$$

where $Q^{\prime}=4 u(b d)^{2}$ and $Q^{\prime \prime}=u^{2}(b d)^{2}-2 \pi u$. Using $u^{2} \leqslant u$ and the lower bound on $\pi$ gives $Q^{\prime \prime} \leqslant 0$. On the other hand, recalling that $u=\frac{1}{2} \beta^{1-p}$, we see that $u \leqslant \frac{1}{6}$ for all $\beta, p \geqslant 2$ such that $(\beta, p) \neq(2,2)$. Hence in this case $Q^{\prime} \leqslant \frac{2}{3}(b d)^{2} \leqslant \frac{8}{3}(a c)^{2} \leqslant$ $3(a c)^{2}$, from which (3.1) follows. In the case where $\beta=p=2$, one has $u=\frac{1}{4}$ and it can be checked that $|\mathrm{RN}(b d)-b d| \leqslant \frac{1}{4} \mathrm{ulp}(b d) \leqslant \frac{u}{2}|b d|$; this implies $Q^{\prime} \leqslant 2(a c)^{2}$ and thus (3.1) follows as well.

If $|a c|<\frac{1}{2}|b d|$ then $|R|=|b d|-|a c|$ and, by Lemma 3.2 and Lemma 3.3,

$$
\begin{aligned}
Q & \leqslant(2|b d|-|a c|)^{2}+(|a d|+2|b c|)^{2} \\
& =(a c)^{2}+4(b d)^{2}+(a d)^{2}+4(b c)^{2},
\end{aligned}
$$

which implies the inequality in (3.1).

3.2. Error bound for algorithm $\mathcal{A}_{2}$. We start with the following lemma, which derives an error bound for the CHT algorithm using the standard model (2.2).

Lemma 3.4. Let $r=a b+c d$ and let $\widehat{r}=C H T(a, b, c, d)$. Then

$$
|\widehat{r}-r| \leqslant\left(2 u+u^{2}\right)|r|+\left(2 u^{2}+2 u^{3}\right)(|a b|+|c d|) .
$$


Proof. Recall from algorithm CHT that $e_{1}=a b-\widehat{w}_{1}$ and $e_{2}=c d-\widehat{w}_{2}$, and apply (2.2) to the five remaining floating-point operations: $\widehat{w}_{1}=a b\left(1+\epsilon_{1}\right), \widehat{w}_{2}=$ $c d\left(1+\epsilon_{2}\right), \widehat{f}=\left(\widehat{w}_{1}+\widehat{w}_{2}\right)\left(1+\epsilon_{3}\right), \widehat{e}=\left(e_{1}+e_{2}\right)\left(1+\epsilon_{4}\right)$, and $\widehat{r}=(\widehat{f}+\widehat{e})\left(1+\epsilon_{5}\right)$ with $\epsilon_{i} \in \mathbb{R}$ such that $\left|\epsilon_{i}\right| \leqslant u$ for $1 \leqslant i \leqslant 5$. This implies

$$
\begin{aligned}
\widehat{r} & =\left(r+\left(\widehat{w}_{1}+\widehat{w}_{2}\right) \epsilon_{3}+\left(e_{1}+e_{2}\right) \epsilon_{4}\right)\left(1+\epsilon_{5}\right) \\
& =\left(\left(1+\epsilon_{3}\right) r+\left(e_{1}+e_{2}\right)\left(\epsilon_{4}-\epsilon_{3}\right)\right)\left(1+\epsilon_{5}\right),
\end{aligned}
$$

using $\widehat{w}_{1}=a b-e_{1}$ and $\widehat{w}_{2}=c d-e_{2}$. Using further $e_{1}=-a b \epsilon_{1}$ and $e_{2}=-c d \epsilon_{2}$, we arrive at

$$
\widehat{r}=\left(\left(1+\epsilon_{3}\right) r+\left(a b \epsilon_{1}+c d \epsilon_{2}\right)\left(\epsilon_{3}-\epsilon_{4}\right)\right)\left(1+\epsilon_{5}\right) .
$$

The latter equality is equivalent to

$$
\widehat{r}-r=\left(\epsilon_{3}+\epsilon_{5}+\epsilon_{3} \epsilon_{5}\right) r+\left(a b \epsilon_{1}+c d \epsilon_{2}\right)\left(\epsilon_{3}-\epsilon_{4}\right)\left(1+\epsilon_{5}\right),
$$

and the conclusion follows from the triangle inequality and the bounds $\left|\epsilon_{i}\right| \leqslant u$.

The error bound for algorithm CHT in Lemma 3.4 can be very pessimistic, at least in radix 2: for example, when $(a, b, c, d)=(N-1, N+1,-N, N)$ and $N=\beta^{p}-1$, it says that the relative error $|\widehat{r} / r-1|$ is upper bounded by a value larger than 1 , while we know from [14] that $|\widehat{r} / r-1| \leqslant 2 u+O\left(u^{2}\right)$ when $\beta=2$. Despite this weakness, it can be used to establish the following result, which gives a normwise relative error bound of the form $2 u+O\left(u^{2}\right)$ for algorithm $\mathcal{A}_{2}$.

Theorem 3.2. For $\beta, p \geqslant 2$ and in the absence of underflow and overflow, algorithm $\mathcal{A}_{2}$ computes $\widehat{z}_{2}$ such that

$$
\left|\widehat{z}_{2}-z\right| \leqslant\left(2 u+6 u^{2}\right)|z| .
$$

Proof. We can assume without loss of generality that $a b c d \geqslant 0$. This implies $\operatorname{sign}(a c)=\operatorname{sign}(b d)$ and $\operatorname{sign}(a d)=\operatorname{sign}(b c)$, and then $|R|=|| a c|-| b d||$ and $|I|=|a d|+|b c|$. Applying Lemma 3.4 twice - first with $r=a c-b d$ and then with $r=a d+b c$, we obtain

$$
\left|\widehat{R}_{2}-R\right| \leqslant 2 u\left(\left(1+\frac{1}{2} u\right)|R|+\left(u+u^{2}\right) \widetilde{R}\right), \quad \widetilde{R}:=|a c|+|b d|,
$$

and

$$
\left|\widehat{I}_{2}-I\right| \leqslant 2 u\left(1+\frac{3}{2} u+u^{2}\right)|I| .
$$

We deduce that $\left|\widehat{z}_{2}-z\right| \leqslant 2 u \sqrt{B}$, where

$$
B=\left(1+\frac{1}{2} u\right)^{2} R^{2}+\left(2 u+3 u^{2}+u^{3}\right)|R| \widetilde{R}+\left(u+u^{2}\right)^{2} \widetilde{R}^{2}+\left(1+\frac{3}{2} u+u^{2}\right)^{2} I^{2} .
$$

Since $R^{2}+I^{2}=|z|^{2}$, we can rewrite $B$ as

$$
B=\left(1+u+\frac{1}{4} u^{2}\right)|z|^{2}+2 u\left(|R| \widetilde{R}+I^{2}\right)+u^{2} B^{\prime},
$$

where

$$
B^{\prime}=(3+u)|R| \widetilde{R}+\left(1+2 u+u^{2}\right) \widetilde{R}^{2}+\left(4+3 u+u^{2}\right) I^{2} .
$$

Now, using the fact that $2 a b c d \leqslant \min \left\{(a c)^{2}+(b d)^{2},(a d)^{2}+(b c)^{2}\right\}$, we can check that $|R| \widetilde{R}$ and $\widetilde{R}^{2}$ and $I^{2}$ are upper bounded by $|z|^{2}=(a c)^{2}+(b d)^{2}+(a d)^{2}+(b c)^{2}$. Hence $B^{\prime} \leqslant\left(8+6 u+2 u^{2}\right)|z|^{2}$ and

$$
\begin{aligned}
B & \leqslant\left(1+5 u+\frac{1}{4} u^{2}\right)|z|^{2}+u^{2} B^{\prime} \\
& \leqslant\left(1+5 u+\frac{33}{4} u^{2}+6 u^{3}+2 u^{4}\right)|z|^{2} \\
& \leqslant(1+3 u)^{2}|z|^{2} \quad \text { for all } u \leqslant 1 / 4 .
\end{aligned}
$$


Consequently, we have shown that $\left|\widehat{z}_{2}-z\right| \leqslant 2 u(1+3 u)|z|$ for all $\beta, p \geqslant 2$.

\section{Asymptotic Optimality of the ERROR BOUndS FOR ALgORIthms $\mathcal{A}_{1}, \mathcal{A}_{2}$, AND $\mathcal{A}_{3}$}

In the previous section we obtained relative error bounds of the form $2 u$ and $2 u+O\left(u^{2}\right)$ for algorithms $\mathcal{A}_{1}$ and $\mathcal{A}_{2}$, respectively; for algorithm $\mathcal{A}_{3}$, we recalled in Section 2.3 that a bound of the form $2 u$ follows as an immediate consequence of [8, Theorem 1.2]. These three upper bounds hold for any $\beta, p \geqslant 2$ and in the absence of underflows and overflows. Here we provide certificates showing that these upper bounds are asymptotically optimal as $u \rightarrow 0$. Each certificate consists of a pair $(a, b) \in \mathbb{F} \times \mathbb{F}$ expressed explicitly in terms of $\beta$ and $p$, and for which we prove that the relative error when evaluating the square $(a+i b)^{2}$ by a given multiplication algorithm is lower bounded by $2 u-O\left(u^{1.5}\right)$. Our proofs require only a mild assumption on the radix and precision, namely $\beta^{p-1} \geqslant 12$ (which is satisfied by all IEEE floating-point formats), and assume as before that underflows and overflows do not occur.

4.1. Certificate for algorithms $\mathcal{A}_{1}$ and $\mathcal{A}_{3}$. We provide in Theorem 4.1 a single certificate that applies to both algorithms $\mathcal{A}_{1}$ and $\mathcal{A}_{3}$, thus proving the asymptotic optimality of their common error bound $2 u$. For this, we first establish the following technical lemma. Here and hereafter, $\lfloor\cdot\rfloor$ denotes the usual floor function.

Lemma 4.1. For $\beta \geqslant 2$ and $p \geqslant 2$, let $n=\left\lfloor\sqrt{\frac{1}{2} \beta^{p-1}}\right\rfloor+1$. Then

(i) $\beta^{p-1}+n \leqslant \beta^{p}$;

(ii) $\beta^{p-1}+2 n<\beta^{p}$ if $\beta^{p-1} \geqslant 7$;

(iii) $\frac{1}{2} \beta^{p-1}<n^{2} \leqslant \beta^{p-1}$ if $\beta^{p-1} \geqslant 12$.

Proof. (i) Since $\beta \geqslant 2$, the announced inequality is implied by $\sqrt{\frac{1}{2} \beta^{p-1}}+1 \leqslant \beta^{p-1}$ or, equivalently, by $2 u+\sqrt{u}-1 \leqslant 0$ with $u=\frac{1}{2} \beta^{1-p}$. The latter inequality holds, since the assumptions $\beta \geqslant 2$ and $p \geqslant 2$ imply $u \leqslant 1 / 4$.

(ii) The claimed inequality is implied by $3 u+\sqrt{u}+\frac{1-\beta}{2} \leqslant 0$. Since $\beta \geqslant 2$ it suffices to ensure $3 u+\sqrt{u}-\frac{1}{2} \leqslant 0$, that is, $\sqrt{u} \leqslant \frac{\sqrt{7}-1}{6}=: v$. The latter inequality is equivalent to $\beta^{p-1} \geqslant \frac{1}{2 v^{2}}=6.64 \ldots$, which is true by assumption.

(iii) By definition $n>\sqrt{\frac{1}{2} \beta^{p-1}}$, hence the lower bound on $n^{2}$. The upper bound is implied by $\frac{1}{\sqrt{2}} \beta^{(p-1) / 2}+1 \leqslant \beta^{(p-1) / 2}$, that is, $\beta^{p-1} \geqslant\left(1-\frac{1}{\sqrt{2}}\right)^{-2}=11.65 \ldots$, which is true by assumption.

Theorem 4.1. Let $a, b \in \mathbb{F}$ be given by

$$
a=\operatorname{pred}\left(\sqrt{\frac{1}{2} \beta^{p-1}}\right), \quad b=\beta^{p-1}+\left\lfloor\sqrt{\frac{1}{2} \beta^{p-1}}\right\rfloor+1,
$$

where, for $t \in \mathbb{R}_{>0}$, $\operatorname{pred}(t)=\max \{f \in \mathbb{F}: f<t\}$ denotes the predecessor of $t$ in $\mathbb{F}$. Let also $\widehat{z}_{1}$ and $\widehat{z}_{3}$ be the approximations to $z=(a+i b)^{2}$ computed by algorithms $\mathcal{A}_{1}$ and $\mathcal{A}_{3}$, respectively. If $\beta^{p-1} \geqslant 12$ then, barring underflow and overflow,

$$
\left|\widehat{z}_{h} / z-1\right|>2 u-8 u^{1.5}-4 u^{2}, \quad h \in\{1,3\} .
$$


Proof. Note first that both $a$ and $b$ are indeed in $\mathbb{F}$ : for $a$ this is true by definition, while for $b$ this is a direct consequence of Lemma 4.1 (i).

Let us first prove the lower bound for algorithm $\mathcal{A}_{1}$. Writing $R$ for the real part of $z$ and $\widehat{R}_{1}$ for the real part of $\widehat{z}_{1}$, we have

$$
\left|\widehat{z}_{1} / z-1\right| \geqslant\left|\widehat{R}_{1}-R\right| /|z| \text {. }
$$

The rest of the proof consists of deriving suitable upper and lower bounds on, respectively, $|z|$ and $\left|\widehat{R}_{1}-R\right|$. These bounds will be expressed in terms of $u=\frac{1}{2} \beta^{1-p}$.

By definition of $a$ we have the strict inequality

$$
a^{2}<\frac{1}{2} \beta^{p-1}=\frac{1}{4} u^{-1}
$$

and, by definition of $b$,

$$
b^{2} \leqslant\left(\frac{1}{2} u^{-1}+\frac{1}{2} u^{-1 / 2}+1\right)^{2}=\frac{1}{4} u^{-2}+\frac{1}{2} u^{-3 / 2}+\frac{5}{4} u^{-1}+u^{-1 / 2}+1 .
$$

Applying (4.2) and (4.3) to $|z|=a^{2}+b^{2}$ thus gives the upper bound

$$
|z|<\frac{1}{4} u^{-2}+\frac{1}{2} u^{-3 / 2}+\frac{3}{2} u^{-1}+u^{-1 / 2}+1
$$

Let us now derive a lower bound on $\left|\widehat{R}_{1}-R\right|$. Defining $s=b^{2}$ and $\widehat{s}=\mathrm{RN}(s)$ we have

$$
\widehat{R}_{1}=\mathrm{RN}\left(a^{2}-\widehat{s}\right) \quad \text { and } \quad R=a^{2}-s .
$$

Furthermore, with $n=\left\lfloor\sqrt{\frac{1}{2} \beta^{p-1}}\right\rfloor+1$, we can write $b=\beta^{p-1}+n$ and then

$$
s=f+g, \quad f=\left(\beta^{p-1}+2 n\right) \beta^{p-1}, \quad g=n^{2} .
$$

Lemma 4.1 (ii) shows that $f$ is in $\mathbb{F}$ and satisfies $\operatorname{ulp}(f)=\beta^{p-1}$. Then, applying Lemma 4.1 (iii) gives $\frac{1}{2} \mathrm{ulp}(f)<g \leqslant u l p(f)$, so that rounding $s$ to nearest produces

$$
\widehat{s}=f+\operatorname{ulp}(f) .
$$

A first consequence of (4.5) is $\beta^{2 p-2}<\widehat{s} \leqslant \beta^{2 p-1}$, which together with (4.2) yields

$$
\widehat{R}_{1}=-\widehat{s},
$$

and then $\left|\widehat{R}_{1}-R\right|=\left|\widehat{s}-s+a^{2}\right|$. Another consequence is that $\widehat{s}-s=u \operatorname{lp}(f)-g$ is nonnegative, which implies further

$$
\left|\widehat{R}_{1}-R\right|=\widehat{s}-s+a^{2} .
$$

Third, it turns out that $\widehat{s}-s$ and $a^{2}$ are large enough to provide a useful lower bound on the relative error. More precisely,

$$
\begin{aligned}
\widehat{s}-s & =\beta^{p-1}-n^{2} \\
& \geqslant \beta^{p-1}-\left(\sqrt{\frac{1}{2} \beta^{p-1}}+1\right)^{2}=\frac{1}{4} u^{-1}-u^{-1 / 2}-1
\end{aligned}
$$

and, since the definition of predecessor implies that $\operatorname{pred}(t) \geqslant t-u \operatorname{lp}(t)$ for $t>0$,

$$
\begin{aligned}
a^{2} & \geqslant\left(\sqrt{\frac{1}{2} \beta^{p-1}}-u \operatorname{up}\left(\sqrt{\frac{1}{2} \beta^{p-1}}\right)\right)^{2} \\
& \geqslant \frac{1}{2} \beta^{p-1}(1-2 u)^{2}=\frac{1}{4} u^{-1}-1+u .
\end{aligned}
$$

From (4.7), (4.8), (4.9), and $u=\frac{1}{2} \beta^{1-p}$ we deduce that

$$
\left|\widehat{R}_{1}-R\right| \geqslant \frac{1}{2} u^{-1}-u^{-1 / 2}-2+u \quad \text { for } \beta^{p-1} \geqslant 12 .
$$


Combining (4.1), (4.4), (4.10) we have, overall,

$$
\left|\widehat{z}_{1} / z-1\right| \geqslant \frac{2 u-4 u^{3 / 2}-8 u^{2}+4 u^{3}}{1+2 u^{1 / 2}+6 u+4 u^{3 / 2}+4 u^{2}}=: \varphi(u) \quad \text { for } \beta^{p-1} \geqslant 12 .
$$

Furthermore, it is easily checked that $\varphi(u) \geqslant 2 u-8 u^{3 / 2}-4 u^{2}$ and that the latter quantity is positive as soon as $\beta^{p-1} \geqslant 10$, which concludes the proof for $\mathcal{A}_{1}$.

Let us now show that the same lower bound on the normwise relative error also holds for the result $\widehat{z}_{3}$ computed by algorithm $\mathcal{A}_{3}$. The reason for this is that $\mathcal{A}_{3}$ produces in this example the same same real part as $\mathcal{A}_{1}$ : since the real part $\widehat{R}_{3}$ of $\widehat{z}_{3}$ is computed using Kahan's algorithm, we have

$$
\widehat{R}_{3}=\mathrm{RN}\left(\widehat{R}_{1}+e\right)
$$

with $e=\widehat{s}-s$, hence using (4.6) we conclude that $\widehat{R}_{3}=-\mathrm{RN}(s)=\widehat{R}_{1}$.

4.2. Certificate for algorithm $\mathcal{A}_{2}$. We provide here a certificate showing that the relative error bound $2 u+6 u^{2}$ obtained for algorithm $\mathcal{A}_{2}$ in Theorem 3.2 is asymptotically optimal. To prove this result, the following lemma will be used.

Lemma 4.2. Let $x \in \mathbb{R}_{\geqslant 0}$ and $y \in \mathbb{R}_{>0}$. If $x<y-\frac{1}{2} \mathrm{ulp}(y)$ then $R N(x)<y$.

Proof. From (2.1) we deduce that $\operatorname{RN}(x) \leqslant x+\frac{1}{2} \mathrm{ulp}(x)$. On the other hand, $0 \leqslant x<y$ and thus ulp $(x) \leqslant \operatorname{ulp}(y)$. Hence $\operatorname{RN}(x) \leqslant x+\frac{1}{2} \operatorname{ulp}(y)<y$.

Theorem 4.2. Let $a, b \in \mathbb{F}$ be given by

$$
a=R D\left(\left(1-\frac{1}{2} \beta^{1-p}\right) \sqrt{\frac{1}{2} \beta^{p-1}}\right), \quad b=\beta^{p-1}+\left\lfloor\sqrt{\frac{1}{2} \beta^{p-1}}\right\rfloor+1,
$$

where, for $t \in \mathbb{R}, R D(t)=\max \{f \in \mathbb{F}: f \leqslant t\}$ denotes rounding down in $\mathbb{F}$. Let also $\widehat{z}_{2}$ be the approximation to $z=(a+i b)^{2}$ computed by algorithm $\mathcal{A}_{2}$. If $\beta^{p-1} \geqslant 12$ then, barring underflow and overflow,

$$
\left|\widehat{z}_{2} / z-1\right|>2 u-8 u^{1.5}-6 u^{2} .
$$

Proof. The proof is organized in the same way as for Theorem 4.1: defining $\widehat{R}_{2}$ as the real part of $\widehat{z}_{2}$ and $R$ as the real part of $z$, we combine the inequality $\left|\widehat{z}_{2} / z-1\right| \geqslant\left|\widehat{R}_{2}-R\right| /|z|$ with a lower bound on $\left|\widehat{R}_{2}-R\right|$ and an upper bound on $|z|$.

For $|z|=a^{2}+b^{2}$, an upper bound is again easily derived: from $u=\frac{1}{2} \beta^{1-p}$ and the definition of $a$ we have

$$
a^{2} \leqslant \frac{(1-u)^{2}}{4} u^{-1}
$$

which together with the upper bound on $b^{2}$ already obtained in (4.3) leads to

$$
|z| \leqslant \frac{1}{4} u^{-2}+\frac{1}{2} u^{-3 / 2}+\frac{3}{2} u^{-1}+u^{-1 / 2}+\frac{1}{2}+\frac{1}{4} u .
$$

(Here, only the rightmost two summands differ from those in (4.4).)

For $\left|\widehat{R}_{2}-R\right|$, however, obtaining a suitable lower bound is now more involved than in Theorem 4.1, essentially because of a more complicated expression for $\widehat{R}_{2}$. Let

$$
s=b^{2}, \quad \widehat{s}=\mathrm{RN}\left(b^{2}\right), \quad e_{1}=a^{2}-\mathrm{RN}\left(a^{2}\right), \quad e_{2}=s-\widehat{s} .
$$

Then $R=a^{2}-s$ and by definition of algorithm $\mathcal{A}_{2}$ the real part of $\widehat{z}_{2}$ satisfies

$$
\widehat{R}_{2}=\operatorname{RN}\left(\operatorname{RN}\left(\operatorname{RN}\left(a^{2}\right)-\widehat{s}\right)+\widehat{e}\right), \quad \widehat{e}=\operatorname{RN}\left(e_{1}+e_{2}\right) .
$$


As already shown in the proof of Theorem 4.1 (where $b$ is the same as here), if $\beta^{p-1} \geqslant 12$ then the floating-point number $\widehat{s}$ satisfies the following:

$$
\begin{gathered}
\hat{s} \geqslant 0, \quad \operatorname{ulp}(\widehat{s}) \geqslant \beta^{p-1}, \quad \widehat{s} \neq \beta^{2 p-2}, \\
\widehat{s}-s \text { is a positive integer such that } \widehat{s}-s<\frac{1}{2} \beta^{p-1} .
\end{gathered}
$$

We will now see that the quantities $\operatorname{RN}\left(a^{2}\right)$ and $|\widehat{e}|$ are smaller than $\frac{1}{2} \mathrm{ulp}(\widehat{s})$, thus implying that the first identity in (4.14) simplifies to $\widehat{R}_{2}=-\widehat{s}$.

Bounding $\mathrm{RN}\left(a^{2}\right)$. From (4.12) we have $a^{2} \leqslant x$ with $x=\frac{1}{2} \beta^{p-1}-\frac{1}{2}+\frac{u}{4}$ and thus, by rounding to nearest, $\mathrm{RN}\left(a^{2}\right) \leqslant \mathrm{RN}(x)$. On the other hand, setting $y=\frac{1}{2} \beta^{p-1}$, we deduce from $\beta \geqslant 2$ that $y$ belongs to $\left[\beta^{p-2}, \beta^{p-1}\right)$ and that $\operatorname{ulp}(y)=\beta^{-1} \leqslant 1 / 2$. Consequently, $x+\frac{1}{2} \mathrm{ulp}(y) \leqslant \frac{1}{2} \beta^{p-1}-\frac{1}{4}+\frac{u}{4}<y$ for $\beta \geqslant 2$ and $p \geqslant 2$. Applying Lemma 4.2 then gives $\mathrm{RN}(x)<y$ and we conclude that

$$
\operatorname{RN}\left(a^{2}\right)<\frac{1}{2} \beta^{p-1} .
$$

- Bounding $|\widehat{e}|$. First, by using the properties of the RN function we see that $|\widehat{e}|=\mathrm{RN}\left(\left|e_{1}+e_{2}\right|\right) \leqslant \mathrm{RN}\left(\left|e_{1}\right|+\left|e_{2}\right|\right)$. Then, recalling (4.12) and by definition of $e_{1}$, we have $\left|e_{1}\right| \leqslant u a^{2} \leqslant \frac{1}{4}(1-u)^{2}$. Third, it follows from (4.15b) that $\left|e_{2}\right|$ is an integer such that $\left|e_{2}\right|<\frac{1}{2} \beta^{p-1}$; since $\beta^{p-1}$ is also an integer, this strict inequality implies $\left|e_{2}\right| \leqslant \frac{1}{2} \beta^{p-1}-\frac{1}{2}$. Therefore, by adding these bounds on $\left|e_{1}\right|$ and $\left|e_{2}\right|$ and by rounding to nearest, we obtain $|\widehat{e}| \leqslant \mathrm{RN}\left(x^{\prime}\right)$ with $x^{\prime}=\frac{1}{2} \beta^{p-1}-\frac{1}{4}-\frac{u}{2}+\frac{u^{2}}{4}$. Taking as in the previous paragraph $y=\frac{1}{2} \beta^{p-1}$, we can check that $x^{\prime}<y-\frac{1}{2} \mathrm{ulp}(y)$, which by Lemma 4.2 implies $\mathrm{RN}\left(x^{\prime}\right)<y$ and then

$$
|\widehat{e}|<\frac{1}{2} \beta^{p-1} \text {. }
$$

From (4.15a) and (4.16) it follows that $\operatorname{RN}\left(\operatorname{RN}\left(a^{2}\right)-\widehat{s}\right)$ equals $-\widehat{s}$, so that $\widehat{R}_{2}=\mathrm{RN}(-\widehat{s}+\widehat{e})$. Applying (4.15a) and (4.17) to the latter identity gives further

$$
\widehat{R}_{2}=-\widehat{s} \text {. }
$$

Since $\widehat{s}-s$ is nonnegative by (4.15a), we deduce that $\left|\widehat{R}_{2}-R\right|=\widehat{s}-s+a^{2}$. Furthermore, the definition of $a$ yields

$$
\begin{aligned}
a^{2} & \geqslant(1-2 u)^{2}\left(1-\frac{1}{2} \beta^{1-p}\right)^{2} \frac{1}{2} \beta^{p-1} \\
& =\frac{1}{4} u^{-1}-\frac{3}{2}+\frac{13}{4} u-3 u^{2}+u^{3},
\end{aligned}
$$

which together with (4.8) leads to

$$
\left|\widehat{R}_{2}-R\right| \geqslant \frac{1}{2} u^{-1}-u^{-1 / 2}-\frac{5}{2}+\frac{13}{4} u-3 u^{2}+u^{3} \quad \text { for } \beta^{p-1} \geqslant 12 .
$$

From (4.13) and (4.18) it follows that if $\beta^{p-1} \geqslant 12$, then the relative error $\left|\widehat{z}_{2} / z-1\right|$ is lower bounded by a rational function $\psi(u)$ which is easily seen to be larger than $2 u-8 u^{3 / 2}-6 u^{2}>0$. This concludes the proof.

It turns out that the certificate introduced in Theorem 4.2 for algorithm $\mathcal{A}_{2}$ can also be used to show that when evaluating $a^{2}-b^{2}$ with CHT or $(a+i b)^{2}$ with $\mathcal{A}_{0}$, then the relative error can have the form $2 u-O\left(u^{1.5}\right)$ :

Corollary 4.1. Given $a, b \in \mathbb{F}$ as in (4.11), let $r=a^{2}-b^{2}$ and $z=(a+i b)^{2}$. Let also $\widehat{r}$ and $\widehat{z}_{0}$ be the approximations to $r$ and $z$ computed by algorithms CHT and $\mathcal{A}_{0}$. If $\beta^{p-1} \geqslant 12$ then, barring underflow and overflow, both $|\widehat{r} / r-1|$ and $\left|\widehat{z}_{0} / z-1\right|$ are larger than $2 u-8 u^{1.5}-6 u^{2}$. 
Proof. For such $a$ and $b$, we have seen in the proof of Theorem 4.2 that the correction term $\widehat{e}$ in (4.14) has no effect on the initial approximation $\widehat{R}_{0}=\operatorname{RN}\left(\operatorname{RN}\left(a^{2}\right)-\right.$ $\left.\operatorname{RN}\left(b^{2}\right)\right)$, so that $\widehat{R}_{2}=\widehat{R}_{0}$. Note also that $\widehat{R}_{0}=\operatorname{Re} \widehat{z}_{0}, \widehat{r}=\widehat{R}_{2}$, and $r=R=\operatorname{Re} z$. Hence $|\widehat{r} / r-1| \geqslant|\widehat{r}-r| /|z|=\left|\widehat{R}_{2}-R\right| /|z|$ and, on the other hand, $\left|\widehat{z}_{0} / z-1\right| \geqslant$ $\left|\widehat{R}_{0}-R\right| /|z|=\left|\widehat{R}_{2}-R\right| /|z|$. In each case the conclusion follows from reusing the fact that (4.13) and (4.18) imply $\left|\widehat{R}_{2}-R\right| /|z|>2 u-8 u^{1.5}-6 u^{2}$.

This corollary shows in particular that the relative error bound $2 u+O\left(u^{2}\right)$ is asymptotically optimal for algorithm CHT in radix $\beta=2$, thus providing an alternative to the proof given in $[14, \S 4]$, which takes $a=c=2^{p}-1, b=2^{p-3}+1 / 2$, and $d=2^{p-3}+1 / 4$ (and breaks ties to an even last bit).

\section{CONCLUSION}

It has been shown that the availability of an FMA instruction makes it possible to improve the normwise relative error in computing a complex floating-point product from a bound of $\sqrt{5} u$ to a bound of $2 u$. We have also shown that the term $2 u$ is best possible not only for the basic algorithm $\mathcal{A}_{1}$, but also for the compensated versions $\mathcal{A}_{2}$ and $\mathcal{A}_{3}$, and even in the particular case of squaring.

The table below summarizes the bounds obtained for these three algorithms as well as those given in [3] for algorithm $\mathcal{A}_{0}$, which makes no use of the FMA; unless otherwise stated, the lower bounds assume $\beta^{p-1} \geqslant 12$ and the upper bounds are valid for any $\beta, p \geqslant 2$.

\begin{tabular}{c|ccccc} 
& lower bound on largest & upper bound on largest & flop & \multicolumn{2}{c}{ properties: } \\
& normwise error & normwise error & count & P1 & P2 \\
\hline $\mathcal{A}_{0}$ & $\sqrt{5} u-O\left(u^{2}\right)$ if $\beta=2$ & $\sqrt{5} u$ if $\beta^{p-1} \geqslant 16$ & 6 & yes & yes \\
$\mathcal{A}_{1}$ & $2 u-8 u^{1.5}-4 u^{2}$ & $2 u$ & 4 & no & no \\
$\mathcal{A}_{2}$ & $2 u-8 u^{1.5}-6 u^{2}$ & $2 u+6 u^{2}$ & 14 & yes & yes \\
$\mathcal{A}_{3}$ & $2 u-8 u^{1.5}-4 u^{2}$ & $2 u$ & 8 & no & yes
\end{tabular}

This table also displays in each case the number of floating-point operations (flops) used, and whether the following two basic properties of complex multiplication are preserved or not:

(P1) $\quad x, y \in \mathbb{C} \quad \Rightarrow \quad x y=y x \quad$ (commutativity);

(P2) $\quad x \in \mathbb{C} \Rightarrow x \bar{x} \in \mathbb{R}$,

where $\bar{x}$ denotes the conjugate of $x$. (Further details showing why these properties are indeed preserved or not can be found in Appendix A.)

If we are only interested in reducing the normwise relative error, just using the conventional algorithm $\mathcal{A}_{1}$ suffices to achieve the error bound $2 u$. When a small componentwise error is needed, then either algorithm $\mathcal{A}_{2}$ or algorithm $\mathcal{A}_{3}$ must be used, since unlike $\mathcal{A}_{0}$ and $\mathcal{A}_{1}$ they ensure high relative accuracy for both the real and imaginary parts of the computed product. If in addition properties $\mathrm{P} 1$ and $\mathrm{P} 2$ are essential, then algorithm $\mathcal{A}_{2}$ is the only choice; otherwise, one might prefer algorithm $\mathcal{A}_{3}$, which is cheaper in terms of flops.

Finally, two further remarks can be made, namely about squaring and division: 
Algorithm $\mathcal{A}_{0}$ and complex squaring. We have seen in Section 4 that the leading error term $2 u$ associated with algorithms $\mathcal{A}_{1}, \mathcal{A}_{2}, \mathcal{A}_{3}$ is already sharp when squaring a complex number, that is, when evaluating $(a+i b)^{2}$ instead of a general product $(a+i b)(c+i d)$. A natural question is whether this is also the case for the bound $\sqrt{5} u$ associated with algorithm $\mathcal{A}_{0}$. The answer is 'no', at least when $\beta=2$ : when squaring a complex number with algorithm $\mathcal{A}_{0}$, the normwise error bound $\sqrt{5} u$ of Brent, Percival, and Zimmermann [3] can be reduced further to $2 u$, and this new bound turns out to be asymptotically optimal; see Appendix B for a detailed proof.

Application to complex division. A direct application of our error bounds is to complex division. As noted by Baudin in $[1, \mathrm{p} .25]$, if the quotient $x / y$ is evaluated using the conventional formula $x / y=(x \bar{y}) /(y \bar{y})$, and if the multiplication algorithm used to evaluate the numerator $x \bar{y}$ has its normwise relative error bounded by $\lambda u+O\left(u^{2}\right)$, then the normwise relative error of division is bounded by $B=(3+\lambda) u+O\left(u^{2}\right)$. Without an FMA, using algorithm $\mathcal{A}_{0}$ gives $\lambda=\sqrt{5}$ and, therefore, $B<5.237 u+O\left(u^{2}\right)$. In contrast, if we use any of the FMA-based algorithms $\mathcal{A}_{1}, \mathcal{A}_{2}, \mathcal{A}_{3}$ then $B=5 u+O\left(u^{2}\right)$.

\section{Appendix A. Properties of COMPlex multiplication}

Complex multiplication satisfies the following two basic properties:

$$
\begin{aligned}
& x, y \in \mathbb{C} \quad \Rightarrow \quad x y=y x \quad \text { (commutativity); } \\
& x \in \mathbb{C} \quad \Rightarrow \quad x \bar{x} \in \mathbb{R},
\end{aligned}
$$

where $\bar{x}$ denotes the conjugate of $x$. The following table indicates whether these properties are preserved or not by the four complex multiplication algorithms considered in this paper:

\begin{tabular}{ccc} 
& P1 & P2 \\
\hline $\mathcal{A}_{0}$ & yes & yes \\
$\mathcal{A}_{1}$ & no & no \\
$\mathcal{A}_{2}$ & yes & yes \\
$\mathcal{A}_{3}$ & no & yes
\end{tabular}

Note first that both properties are clearly preserved by algorithm $\mathcal{A}_{0}$ : for all $a, b, c, d \in \mathbb{F}$, we have $\mathcal{A}_{0}(a+i b, c+i d)=\mathcal{A}_{0}(c+i d, a+i b)$ and $\mathcal{A}_{0}(a+i b, a-i b) \in \mathbb{F}$ simply because because addition and multiplication over $\mathbb{R}$ are commutative operations. Algorithm $\mathcal{A}_{2}$ satisfies property $\mathrm{P} 1$ for the same reason; it also satisfies $\mathrm{P} 2$ because $\mathrm{RN}(-t)=-\mathrm{RN}(t)$ for all real $t$.

Property P2 is lost for algorithm $\mathcal{A}_{1}$, since $\mathcal{A}_{1}(a+i b, a-i b)$ has its imaginary part equal to $\mathrm{RN}(\mathrm{RN}(a b)-a b)$, which is in general nonzero. On the other hand, we know from $[8$, Theorem 1.2] that $\operatorname{Kahan}(a,-b, b, a)$ returns zero, which implies that $\mathrm{P} 2$ is preserved by algorithm $\mathcal{A}_{3}$.

Finally, property $\mathrm{P} 1$ is lost for both algorithms $\mathcal{A}_{1}$ and $\mathcal{A}_{3}$. Indeed, there exist floating-point numbers $a, b, c, d$ such that $\mathrm{RN}(a b+\mathrm{RN}(c d)) \neq \mathrm{RN}(\mathrm{RN}(a b)+c d)$ and $\operatorname{Kahan}(a, b, c, d) \neq \operatorname{Kahan}(c, d, a, b)$, as illustrated by the following example. 
Example A.1. Let $a, b, c, d \in \mathbb{F}$ defined by

$$
a=\beta^{p-1}, \quad b=c=a+1, \quad d= \begin{cases}\beta^{p}-\frac{\beta}{2} & \text { if } \beta \text { is even } \\ \beta^{p}-\frac{\beta+1}{2} & \text { if } \beta \text { is odd }\end{cases}
$$

Assuming $p \geqslant 3$, it can be checked that $\mathrm{RN}(a b+\mathrm{RN}(c d))$ and $\operatorname{Kahan}(a, b, c, d)$ are both equal to $\beta^{2 p-1}+\beta^{2 p-2}$, while $\operatorname{RN}(\operatorname{RN}(a b)+c d)$ and $\operatorname{Kahan}(c, d, a, b)$ are both equal to $\beta^{2 p-1}+\beta^{2 p-2}+\beta^{p}$. In this example, a tie occurs when rounding $a b+\mathrm{RN}(c d)$ to nearest, and the standard 'round to the nearest even' tie-breaking rule (roundTiesToEven rounding attribute in [7]) is then used.

\section{Appendix B. AcCuracy of Squaring with algorithm $\mathcal{A}_{0}$ IN RAdix 2}

When evaluating the complex square $z=(a+i b)^{2}$ with $a, b$ in $\mathbb{F}$, algorithm $\mathcal{A}_{0}$ returns $\widehat{z}_{0}=\widehat{R}_{0}+i \widehat{I}_{0}$ with $\widehat{R}_{0}=\operatorname{RN}\left(\operatorname{RN}\left(a^{2}\right)-\operatorname{RN}\left(b^{2}\right)\right)$ and $\widehat{I}_{0}=\operatorname{RN}(2 \operatorname{RN}(a b))$. If $\beta=2$ then $\widehat{I}_{0}=2 \mathrm{RN}(a b)$, so that only one rounding error is committed when evaluating the imaginary part. The theorem below shows that in this special case the bound $\sqrt{5} u$ of Brent, Percival, and Zimmermann [3] can be reduced to $2 u$.

Theorem B.1. Assume $\beta=2$ and, given $a$ and $b$ in $\mathbb{F}$, let $z=(a+i b)^{2}$. Then, for $p \geqslant 2$ and in the absence of underflow and overflow, algorithm $\mathcal{A}_{0}$ computes $\widehat{z}_{0}$ such that

$$
\left|\widehat{z}_{0}-z\right| \leqslant 2 u|z|
$$

and this relative error bound is asymptotically optimal.

Proof. Note first that if $a$ and $b$ are swapped, then only the signs of the real (exact and computed) parts change, so the error $\left|\widehat{z}_{0}-z\right|$ remains the same. Hence we can assume $a^{2} \geqslant b^{2}$. Defining $s=\mathrm{RN}\left(a^{2}\right)-\mathrm{RN}\left(b^{2}\right)$, we have

$$
\left|\widehat{R}_{0}-R\right| \leqslant|\mathrm{RN}(s)-s|+\left|\mathrm{RN}\left(a^{2}\right)-a^{2}\right|+\left|\mathrm{RN}\left(b^{2}\right)-b^{2}\right| .
$$

If $s \in \mathbb{F}$ then (B.1) gives $\left|\widehat{R}_{0}-R\right| \leqslant u\left(a^{2}+b^{2}\right)$. Since $\left|\widehat{I}_{0}-I\right| \leqslant 2 u|a b|$ and $|z|^{2}=\left(a^{2}+b^{2}\right)^{2}$, it follows that

$$
\begin{aligned}
\left|\widehat{z}_{0}-z\right|^{2} & \leqslant u^{2}\left(a^{4}+6 a^{2} b^{2}+b^{4}\right) \\
& \leqslant 2 u^{2}|z|^{2} .
\end{aligned}
$$

If $s \notin \mathbb{F}$ then, since $a^{2} \geqslant b^{2}$ leads to $\operatorname{RN}\left(a^{2}\right) \geqslant \mathrm{RN}\left(b^{2}\right) \geqslant 0$ and $0 \leqslant s \leqslant \operatorname{RN}\left(a^{2}\right)$, we have $s<\operatorname{RN}\left(a^{2}\right)$. This strict inequality implies ulp $(s) \leqslant \mathrm{ulp}\left(a^{2}\right)$. Hence (B.1) now gives $\left|\widehat{R}_{0}-R\right| \leqslant u\left(2 a^{2}+b^{2}\right)$, so that

$$
\begin{aligned}
\left|\widehat{z}_{0}-z\right|^{2} & \leqslant u^{2}\left(4 a^{4}+8 a^{2} b^{2}+b^{4}\right) \\
& \leqslant 4 u^{2}|z|^{2} .
\end{aligned}
$$

Thus, in both cases we have $\left|\widehat{z}_{0}-z\right| \leqslant 2 u|z|$.

The asymptotic optimality of this bound follows from Corollary 4.1, which says that for $\beta=2$ and $p \geqslant 5$, there exist $a, b$ in $\mathbb{F}$ such that $\left|\widehat{z}_{0} / z-1\right|$ is lower bounded by $2 u-8 u^{1.5}-6 u^{2}$. 


\section{REFERENCES}

1. Michael Baudin, Error bounds of complex arithmetic, June 2011, available at http://forge. scilab.org/upload/compdiv/files/complexerrorbounds_v0.2.pdf.

2. Sylvie Boldo, Pitfalls of a full floating point proof: example on the formal proof of the Veltkamp/Dekker algorithms, Proceedings of Third International Joint Conference on Automated Reasoning (Seattle,Washington), 2006, pp. 52-66.

3. Richard Brent, Colin Percival, and Paul Zimmermann, Error bounds on complex floating-point multiplication, Mathematics of Computation 76 (2007), 1469-1481.

4. Marius Cornea, John Harrison, and Ping Tak Peter Tang, Scientific computing on Itanium ${ }^{\circledR}$. based systems, Intel Press, Hillsboro, OR, 2002.

5. T. J. Dekker, A floating-point technique for extending the available precision, Numer. Math. 18 (1971), 224-242.

6. Nicholas J. Higham, Accuracy and Stability of Numerical Algorithms, second ed., SIAM, Philadelphia, PA, USA, 2002.

7. IEEE Computer Society, IEEE standard for floating-point arithmetic, IEEE Standard 754-2008, August 2008, available at http://ieeexplore.ieee.org/servlet/opac?punumber= 4610933.

8. Claude-Pierre Jeannerod, Nicolas Louvet, and Jean-Michel Muller, Further analysis of Kahan's algorithm for the accurate computation of $2 \times 2$ determinants, Mathematics of Computation 82 (2013), 2245-2264.

9. William Kahan, Further remarks on reducing truncation errors, Comm. ACM 8 (1965), no. 1, 40.

10. Seppo Linnainmaa, Analysis of some known methods of improving the accuracy of floatingpoint sums, BIT 14 (1974), 167-202.

11. _ Software for doubled-precision floating-point computations, ACM Trans. Math. Software 7 (1981), no. 3, 272-283.

12. Ole Møller, Note on quasi double-precision, BIT 5 (1965), 251-255.

13. Q Quasi double-precision in floating point addition, BIT 5 (1965), 37-50.

14. Jean-Michel Muller, On the error of computing $a b+c d$ using Cornea, Harrison and Tang's method, Available at http://hal-ens-lyon.archives-ouvertes.fr/ensl-00862910, September 2013.

15. Jean-Michel Muller, Nicolas Brisebarre, Florent de Dinechin, Claude-Pierre Jeannerod, Vincent Lefèvre, Guillaume Melquiond, Nathalie Revol, Damien Stehlé, and Serge Torres, Handbook of Floating-Point Arithmetic, Birkhäuser Boston, 2010, ACM G.1.0; G.1.2; G.4; B.2.0; B.2.4; F.2.1., ISBN 978-0-8176-4704-9.

16. Michèle Pichat, Correction d'une somme en arithmétique à virgule flottante, Numer. Math. 19 (1972), 400-406.

17. Contributions à l'etude des erreurs d'arrondi en arithmétique à virgule flottante, Ph.D. thesis, Université Scientifique et Médicale de Grenoble, Grenoble, France, 1976.

INRIA, LABoratoire LIP (CNRS, ENS de Lyon, INRIA, UCBL), Université de Lyon 46, allée d'Italie 69364 Lyon CEDex 07, France

E-mail address: claude-pierre.jeannerod@ens-lyon.fr

University of Southern Denmark, Department of Mathematics and Computer Science - Campusvej 55 DK-5230 Odense M Denmark

E-mail address: kornerup@imada.sdu.dk

UCBL, LABORATOIRE LIP (CNRS, ENS de Lyon, INRIA, UCBL), Université de LyON 46, allée d'Italie 69364 Lyon cedex 07, France

E-mail address: nicolas.louvet@ens-lyon.fr

CNRS, LABORATOIRE LIP (CNRS, ENS de Lyon, INRIA, UCBL), Université de Lyon 46, allée d'Italie 69364 Lyon Cedex 07, France

E-mail address: jean-michel.muller@ens-lyon.fr 